\title{
Immunologic Response of Infants to Combined Inactivated Measles-Poliomyelitis Vaccine ${ }^{* * *}$
}

\author{
By \\ Gordon C. Brown* \\ With 2 Figures
}

In previous studies of inactivated poliomyelitis vaccine given to young infants, whether alone or in combination with other antigens such as diphtheria, tetanus and pertussis, the suppressive effect of maternal antibodies on active immunization has been repeatedly described. Inactivated measles vaccine, however, has been studied primarily in preschool or school children and very little information is available concerning the serologic response of young infants to it alone or combined with other antigens. The purpose of this study was to determine the serologic response of 3 and 5 months old infants to a combined inactivated measles-poliomyelitis vaccine.

\section{Experimental}

The vaccine employed was an experimental preparation furnished by the Pfizer Laboratories. The primary series consisted of three doses of $0.5 \mathrm{ml}$ each injected intramuscularly at intervals of two months. The selection of this schedule was chosen on the basis of previous studies by the author which showed it to be superior to a one month interval between injections during the primary series. The study was carried out in the Well Baby Clinics of two hospitals in Ann Arbor. Forty-two infants received their vaccine at the ages of 3,5 , and 7 months, and 23 infants were injected at 5, 7, and 9 months of age. Fifty-two of these 65 children were given a booster injection at the age of 18 months, 11 and 9 months after the primary series respectively, depending upon the schedule. At this time, 9 children received an injection of live attenuated measles vaccine and of killed poliomyelitis vaccine rather than the original com-

* Professor of Epidemiology, School of Public Health, The University of Michigan, Ann Arbor, Michigan, U.S.A.

** Aided by a grant from the National Foundation.

Archiv f. Virusforschung, Bd. XVI, H. 1-5 
bined preparation. Blood specimens were taken before and one month after the primary series, and before and two weeks after booster. In addition, a smail number of children were bled again 6 months following booster. Neutralizing antibodies for both viruses were determined in tissue culture tests* and, in addition, fluorescent antibody determinations were made using the indirect method.

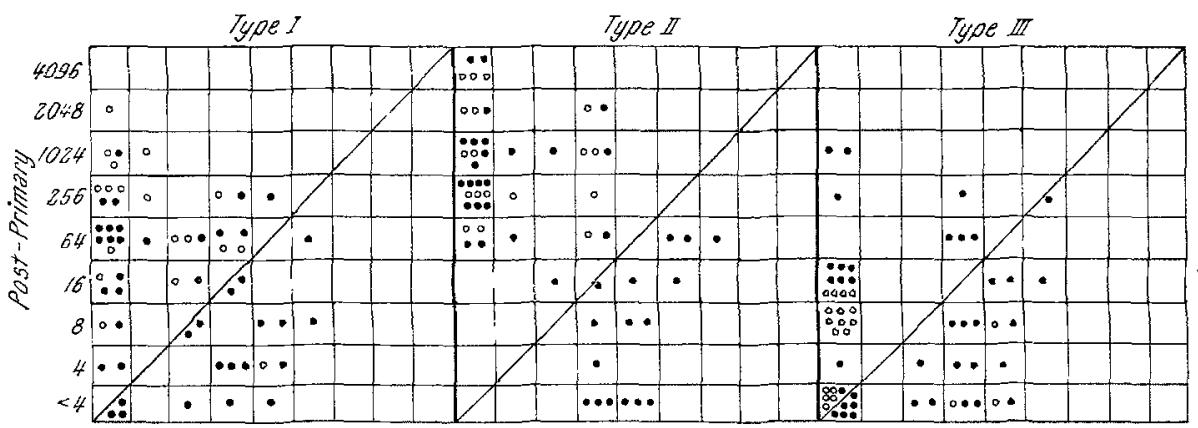

- = Injected at 3,5,7 Months Pre-Primary o = Injected at 5,7,9 Mosths

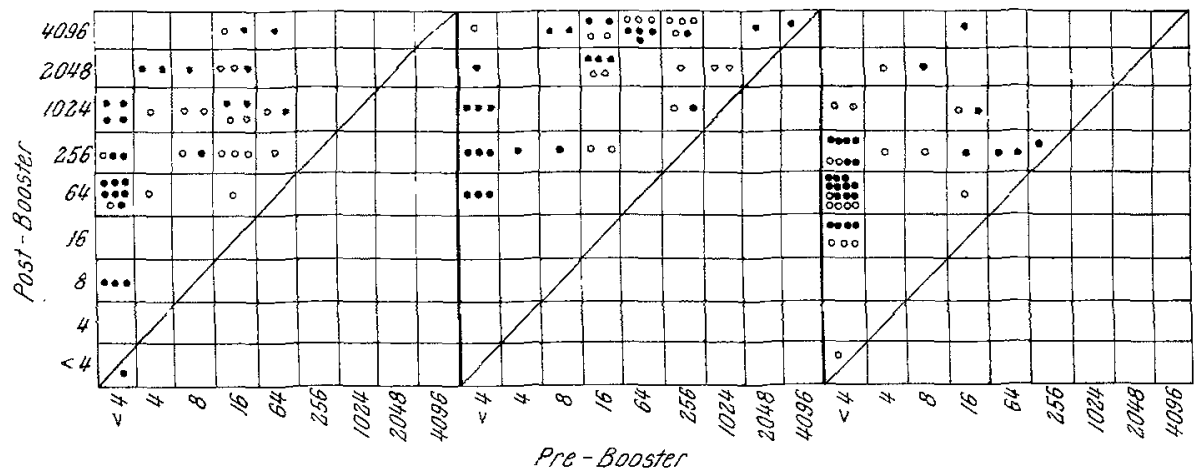

Fig. 1. Poliomyelitis neutralizing antibody titers in infants following combined inactivated measles-poliomyelitis raccine.

\section{Reactions}

Forty-seven of the infants in one clinic were followed for reactions to the vaccine by having the mothers report on specific reactions. Twenty infants showed fussiness, 16 had slight fever, or warmness, 4 lacked appetite and 6 showed slight or moderate local reactions at the site of injection. Many of the reactions followed the second dose of vaccine. The 9 infants who received live, attenuated vaccine for their booster were examined by a physician daily from the 5th through the 10th day following injection. These results are discussed later.

* Neutralization tests for measles virus were performed in the laboratories of Chas. Pfizer and Co. in Terre Haute. Indiana. 


\section{Results}

The neutralizing antibody titers for poliomyelitis before and after both primary and booster immunization are shown in Fig. 1 . It can be seen that many infants retained maternal antibodies at the third month of life and most of these failed to respond to primary vaccination with increased antibody titers. This confirms previous observations of the suppressive effect of passive antibodies on active immunization. In contrast, very few infants had maternal antibodies in high titer at 5 months of life illustrating the importance of the two additional months in allowing further passive antibody decay. Most infants with maternal antibodies in low or in nondetectable titer responded with antibody production although the type III antigen appeared to be the weakest of the 3 types. No apparent difference existed in the response of infants in the two age groups if uncomplicated by the presence of maternal antibodies. The serologic response to booster inoculation was unusually good when compared with previous studies which showed that suppression of Table 1. Primary response of infants to measles-poliomyelitis vaccine primary immunization fre-

\begin{tabular}{|c|c|c|c|}
\hline \multirow{2}{*}{ Age (month) } & \multicolumn{2}{|c|}{ Pre-primary } & \multirow{2}{*}{$\begin{array}{c}\text { Increase } \\
\text { antibody titer }\end{array}$} \\
\hline & Titer & $\mathrm{Nb}$. & \\
\hline \multirow[t]{2}{*}{3} & $<6$ & 12 & 10 \\
\hline & 6 or $>$ & 30 & 3 \\
\hline \multirow[t]{2}{*}{5} & $<6$ & 14 & 14 \\
\hline & 6 or $>$ & 9 & 0 \\
\hline
\end{tabular}
quently resulted in unsatisfactory response to booster injections. In the present study only two infants were left without detectable antibodies following this stimulus. Furthermore, most of the titers attained following booster were quite high suggesting excellent protection against the disease.

Thirty of the 42 infants $(71 \%$ ) receiving their first injection at the age of 3 months had maternal antibodies for measles virus in titers of 1:6 or greater. (Table 1.) Only 3 of these responded to the primary series with increased titers. In contrast 10 of the 12 infants with titers of less than $1: 6$ responded. Only 9 of 23 infants $(39 \%)$ injected first at the age of 5 months still had passive antibodies of 1:6 or greater and none of these responded. In contrast, all 14 with lower titers showed an increase. Thus, $60 \%$ of all the infants receiving vaccine at the age of 5 months responded immunologically in contrast to only $28 \%$ of the total number started two months earlier. The significance of passive antibody suppression of primary response is illustrated by the fact that two of these non-responding children devieloped clinical measles, 6 weeks and 5 months respectively, following the third injection of the primary series.

Fig. 2 shows the neutralizing antibody titers for measles virus before and after primary and secondary immunization. Twenty-four of the 
52 children bled at the time of booster showed retention of antibodies, some in high titer, 9 and 11 months after the primary series. This number might well have been considerably higher but for the fact that 16 of the $28(57 \%)$ without antibodies at this time were non-responders to primary series by virtue of maternal antibody suppression and did not have antibodies to retain.

The response to booster injection must be considered as highly satisfactory since only 2 children failed to show increased antibodies. Both of these, incidently, were non-responders to the primary series. In general

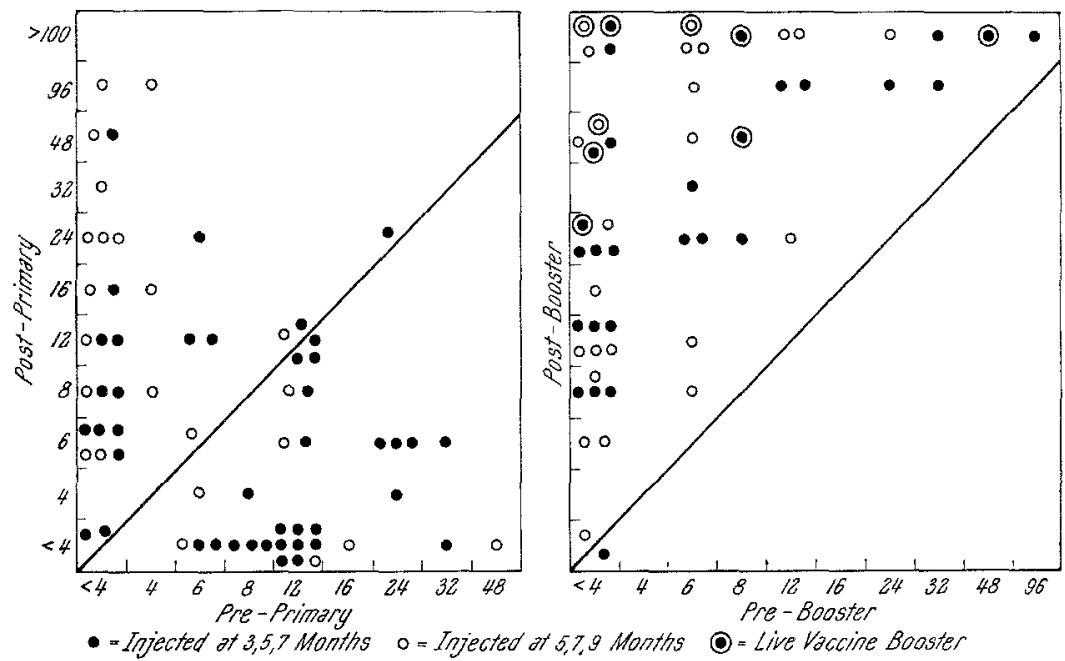

Fig. 2. Measles neutralizing antibody titers in infants following combined inactivated measles-poliomyelitis vaccine.

the titers of antibody in post-booster sera were found to be high. No apparent difference in titers was detected between those children who had received their first injection at the age of 3 months or at 5 months.

The 9 children who were given live, attenuated virus vaccine for their boosters responded with titers very similar to those found in children receiving killed vaccine. Table 2 presents the individual antibody titers detected in these individuals together with the reactions seen. In 4 of the 9, an elevated temperature was recorded, one had a moderate local reaction at the site of inoculation, another, a runny nose, and still another showed a transient rash and otitis media. Five were perfectly normal throughout the period of observation. On the basis of serologic and clinical response to booster it seems unlikely that either of the 2 children thought by their private physicians to have had measles following primary series were in fact accurately diagnosed. 
An interesting and potentially useful corollary to this study was the examination of all sera by the indirect method of fluorescent microscopy. Most specimens examined for poliomyelitis antibodies showed considerable evidence of heterologous reactions between the three types suggesting strongly that this technique is disease, rather than type, specific. On the other hand, very close agreement was observed between the measles neutralizing and fluorescent antibody titers demonstrating that this procedure can be employed equally well for diagnosis and for research.

Table 2. Serologic and clinical history of infants receiving live attenuated measles vaccine booster following killed vaccine primary series

\begin{tabular}{|c|c|c|c|c|c|}
\hline & \multicolumn{4}{|c|}{ Antibody titer } & \multirow[b]{2}{*}{ Reactions after booster } \\
\hline & 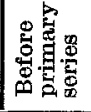 & 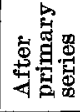 & 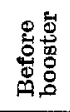 & 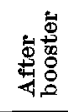 & \\
\hline F. L. & $<8$ & $<4$ & $<4$ & 56 & Moderate fever $5^{* *}, 6,7,8$ \\
\hline K. E. & $<4$ & 6 & 9 & 56 & Slight fever $6,7,8 ; 2+\operatorname{local} 8,9,10$ \\
\hline D. W & $<8$ & $<4$ & *56 & 150 & Slight fever 5 ; runny nose 5,6 \\
\hline T. B. & $<4$ & 12 & $<4$ & 150 & High fever $5,6,7,8$; rash 7 ; otitis media 8 \\
\hline S. H. & $<4$ & 48 & 6 & 100 & None \\
\hline R. J. & 6 & 6 & $<4$ & 100 & None \\
\hline D. C. & $<8$ & 7 & 10 & 100 & None \\
\hline C. L. & 16 & $<4$ & $<4$ & 75 & None \\
\hline S.L. & 12 & 12 & $*<4$ & 28 & None \\
\hline
\end{tabular}

* Clinical measles?

** Numbers indicate day.

\section{Summary}

The serologic response of young infants to combined, inactivated measles-poliomyelitis vaccine is described. Maternal antibodies to both diseases were present in many children at the age of 3 months and in some at the age of 5 months and when present, suppressed the primary response to vaccine. Booster injection of vaccine at the age of 18 months was successful in stimulating increased antibody titers in $96 \%$ of all the children. Booster doses of live attenuated measles virus instead of killed vaccine stimulated the production of comparable titers of antibody. 